\title{
Fast X-ray variability of radio galaxy M87
}

\section{Ryo Imazawa, ${ }^{a, *}$ Yasushi Fukazawa, ${ }^{a}$ Hiromitsu Takahashi ${ }^{a}$ and Mahito Sasada ${ }^{b}$}

${ }^{a}$ Hiroshima University Graduate School of Advanced Science and Engineering, 1-3-1 Kagamiyama, Higashi-Hiroshima, Hiroshima 739-8526, Japan

${ }^{b}$ Hiroshima University, Hiroshima Astrophysical Science Center, 1-3-1 Kagamiyama, Higashi-Hiroshima, Hiroshima 739-8526, Japan

E-mail: imazawa@astro.hiroshima-u.ac.jp

M87 is a radio galaxy and it was detected in the TeV gamma-ray band, but its emission and particle acceleration region are still under debate. We searched for fast X-ray variability of M87 by using X-ray archive data with a long exposure than $10 \mathrm{ksec}$. As a result, we found an intraday variability during Suzaku/XIS data in 2006. Suzaku/XIS cannot resolve each component in the jet, but HST-1 knot standing at $\sim 100$ pc from the core was the brightest component in the X-ray band in this period. Therefore, this variability is likely attributed to HST-1. A soft photon index of 2.38 in the X-ray band indicates that variability component is synchrotron emission by accelerated electrons in HST-1. Assuming the decay time scale as correspond to synchrotron cooling time, we estimated a magnetic field strength to $B \sim 1.94 \delta^{1 / 3} \mathrm{mG}$, which is enough to accelerate particles up to $\mathrm{TeV}$, where $\delta$ is a Doppler factor. In addition, we also found a possible variability of the core during the Chandra/HRC observation in 2017. In this period, a NuStar X-ray spectrum was represented by a power law with a photon index of 1.96. Therefore we could not distinguish this fast variability is due to synchrotron emission or disk/corona emission. From these results, both of the core and HST-1 are could be the origin of the X-ray variability, and we found that knot HST-1 is a candidate of the location of particle acceleration up to TeV.

$37^{\text {th }}$ International Cosmic Ray Conference (ICRC 2021)

July 12 th - 23rd, 2021

Online - Berlin, Germany

\footnotetext{
*Presenter
} 


\section{Introduction}

Radio galaxy is one type of Active Galactic Nuclei (AGN), which has a strong jet and large inclination. Through past TeV gamma-ray observations, 6 radio galaxies were detected and M87 is one of them. M87 is FR-I type radio galaxy and it is known as the nearby radio galaxy ( $z \sim 0.0042$ ;16 Mpc). Its jet components have been resolved by radio, optical, and X-ray observations. Thus, it is a good object sample to study particle accelerations in the jet, thus many observations by each wavelength were performed. TeV gamma-ray from M87 was detected by MAGIC and H.E.S.S., and they found fast variability ( $\sim$ day). It indicates particle acceleration in the jet, but its location is still under debate.

In this study, we studied X-ray fast variability of M87 to get knowledge of the particle acceleration location. We describe observations and data analysis, results of light curves and spectral analysis, and the discussion about the origin of fast X-ray time variability in Section 2-4.

\section{Observation and Analysis}

We chose long exposure ( $>10 \mathrm{ksec}$ ) data to search for X-ray fast variabilities. For Chandra data, we chose data taken by Advanced CCD Imaging Spectrometer (ACIS) and High Resolution Camera (HRC). These imaging observations were performed without grating. For ACIS data, we chose data taken in the short frame time $(0.4 \mathrm{sec}$; sub-array mode) to minimize pile up effects. NuSTAR performed 6 observations with more than $10 \mathrm{ksec}$ between 2017 and 2019, and Suzaku performed an observation in 2006. Thus, there are 15 Chandra data (13 ACIS and 2 HRC), 6 NuSTAR data, and 1 Suzaku data. Each of NuSTAR observations were performed simultaneously with Chandra. Since NuSTAR cannot resolve each jet component, Chandra data were useful and thus we analyzed these data, even though their exposure time is short. We summarized the list of observation data used in this study in the Figure 1.

\subsection{Light curve}

At first, we analyzed X-ray light curves of in each observation to study variability. We derived light curves of each component in the jet: core, HST-1, knot D, and knot A, in Chandra analysis. The extraction region of each component is shown in Figure 2. HST-1, knot D and A are defined in order from the core, and their positions are set at 0.8, 2.9 and 12.4 arcsec from the core, respectively.

Chandra light curves were created by the dmextract command in CIAO in the energy range of 2.0-10.0 keV (HRC data was not filtered) with a time bin of $3600 \mathrm{sec}$ with circular region radius of 2.0 arcsec. To evaluate the variability of the background signal, light curves of the background region are created in the same way to source light curves. The position is at $5.0 \operatorname{arcsec}$ from the core in the direction opposite to the jet.

For NuSTAR, we analyzed light curves of FPMA and FPMB separately in each observation. The source area is defined as a circle with a radius of 30 arcsec. A light curve was created with the lcurve command in an energy range of 8.0-30.0 keV with a time bin of $3600 \mathrm{sec}$. Light curves of the background region were made with the same radius as the source region at 3.2 arcmin from M87. 


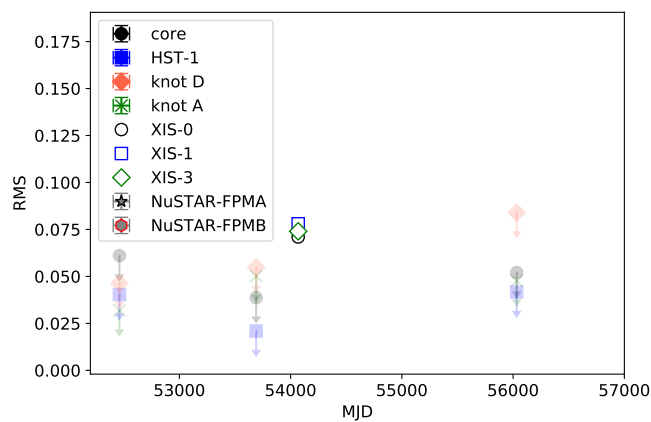

(a)

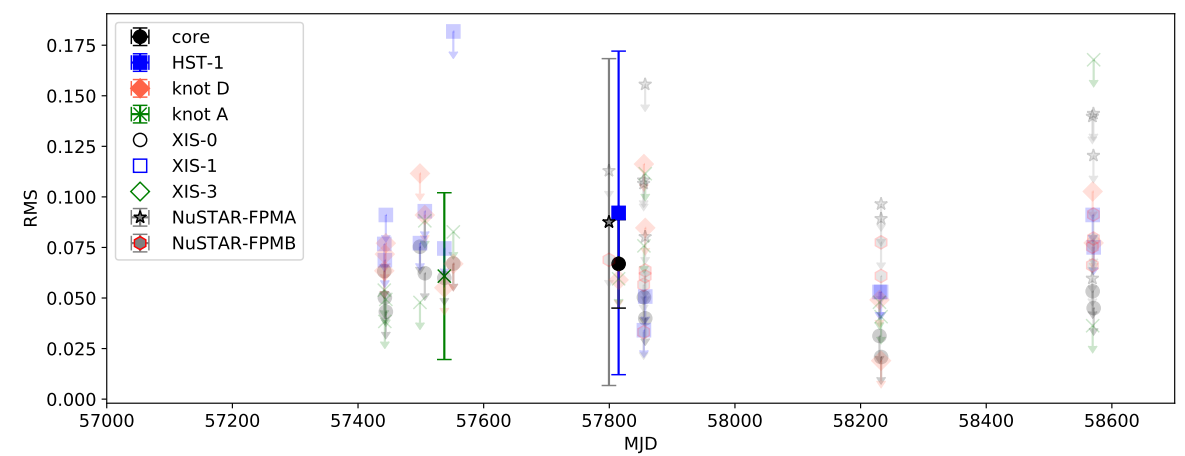

(b)

Figure 1: (a) Observation time and Root Mean Square of X-ray observations analyzed in this study up to 2014 and (b) after 2014. Core, HST-1, knot-D and knot-A represent data observed by Chandra. Each rms error calcurated by propagation of statistical errors in 2 sigma and upper limits were calcurated under the assumption that the statistical error equal to 0 . Upper limits are plotted with clear markers.

The Suzaku observed M87 for two days from November 29 to December 1, 2006 (MJD: 54068-54070). We used data obtained by X-ray Imaging Spectrometer (XIS), onboard the Suzaku. In this observation, XIS consists of three available CCDs; XIS0, XIS1, and XIS3. XIS0 and XIS3 are front-illuminated CCDs, wheareas XIS1 is a back-illuminated CCD. Therefore, the sensitivity in the low energy range of XIS1 is superior to XIS0 and XIS3 [4]. In each instrument, source regions were defined as a circle with a radius of $3.0 \mathrm{arcmin}$ (Fig. 3). The light curves of XIS were created with lcurve command in an energy range of 3.0-10.0 keV with a time bin of $8400 \mathrm{sec}$. For background regions, circles with a radius of $3.0 \mathrm{arcmin}$ at $8.0 \mathrm{arcmin}$ from the core were defined.

\subsection{Spectral analysis}

\subsubsection{NuSTAR and Chandra}

There are six observations taken by both NuSTAR and Chandra simultaneously. We performed simultaneous fitting of NuSTAR and Chandra spectra to obtain the photon index and flux of each component, following the method of [8]. In the simultaneous fitting, three Chandra spectra; core, HST-1, and jet and two NuSTAR spectra; FPMA and FPMB were fitted. The model of hot gas emission was considered for the NuSTAR spectrum, and each power-law model was considered 
for the NuSTAR and Chandra spectrum of each component. Power-law parameters were common between NuSTAR and Chandra.

NuSTAR spectra were extracted in a circular region with a radius of 30 arcsec, and background regions were taken at 80-130 arcsec from M87. For Chandra spectra of each component, the source region of core and HST- 1 were defined as a circle with a radius of $0.4 \mathrm{arcsec}$ and a position specified in Figure 2. For the background of core and HST-1 spectra, a half-circular band region at 2-4 arcsec was specified in the direction opposite to the jet. The jet area, including knots D and A, is defined as shown in Figure 2. The background region was specified as $19.5 \operatorname{arcsec} \times 3 \operatorname{arcsec}$ boxes on both sides of the jet. Chandra spectra were created with specextract command and each spectra were binned so that 1 bin contained more than 20 photons.

The spectral model for simultaneous fit consists of two temperature thermal plasma and three power-law models with Galactic absorption: wabs* (vapec+vapec+pegpwrlw+pegpwrlw+pegpwrlw) in XSPEC. The normalization of vapec was set to 0 for Chandra spectra, and vapec parameters for NuSTAR were fixed to values obtained by Chandra analysis. A column density of Galactic absorption was fixed to $1.94 \times 10^{20} \mathrm{~cm}^{-2}$. Three pegpwrlw models represent emission from the core, HST-1, and jet. Thus, for each Chandra spectrum of the core, HST-1, and jet, only one pegpwrlw normalization was left free and other normalizations were set to 0 . For these analyses, photon index and normalization were unified between NuSTAR and Chandra. Considering cross-calibration uncertainly between FPMA and FPMB of NuSTAR, constant model was added.

\subsection{Suzaku}

We extracted spectral of Suzaku/XIS for the source region with a radius of 3 arcmin (Figure 3), and xisarfgen andxisrmfgen command to make response files.

Spectra were fitted in 1.0-10.0 keV with wabs*(vapec+vapec+pegpwrlw). the abundance parameters of other metals are unified between the two vapec models, but the metal abundances of $\mathrm{H}$, $\mathrm{C}$, and $\mathrm{N}$ were fixed to 1 solar. As a result, we obtained temperatures 2.45 and $1.53 \mathrm{keV}$. The spectra were well fitted with a reduced $\chi$ square of $\chi^{2} /$ d.o.f $=1.25$. Photon index became $\gamma=2.38_{-0.04}^{+0.07}$ (2.0-10.0 keV), and the power-law flux became $2.71_{-0.18}^{+0.18} \times 10^{-11} \mathrm{erg} \mathrm{cm}^{-2} \mathrm{~s}^{-1}$ in $2.0-10.0 \mathrm{keV}$.

\section{Result}

We calculated a Root Mean Square from X-ray light curves of each observation, to evaluate a fast variability. RMS is defined as the following equation [5].

$$
\mathrm{RMS}=\sqrt{\frac{\sum_{i}\left[\left(d_{i}-\bar{d}\right)^{2}-e_{i}^{2}\right]}{N \bar{d}^{2}}}
$$

Where $d_{i}$ is a count rate of $i$-th data, $N$ is the number of data points, $\bar{d}$ is an average, and $e_{i}$ is a statistical error of $d_{i}$. We summarized the RMS values of each observation in Figure 1. The RMS of observations with $\sum_{i}\left[\left(d_{i}-\bar{d}\right)^{2}-e_{i}^{2}\right]<0$, indicating no variability is not shown. As a result, some data showed significant variability. Figure 4(a) and 4(b) shows light curves with significant variability. 


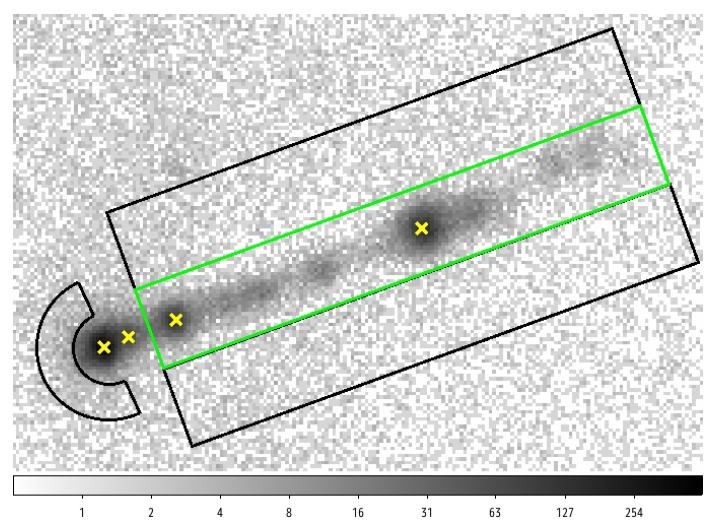

Figure 2: M87 X-ray image obtained via Chandra-HRC (2017-3-02; ID18612). Crosses in the figure show positions of core, HST-1, knot D, and knot A from the left. The green box represents the jet region. The black half-circular band shows the background region of the core and HST-1 and the black boxes show the background region of the jet (including knots D and A) for spectral analysis.
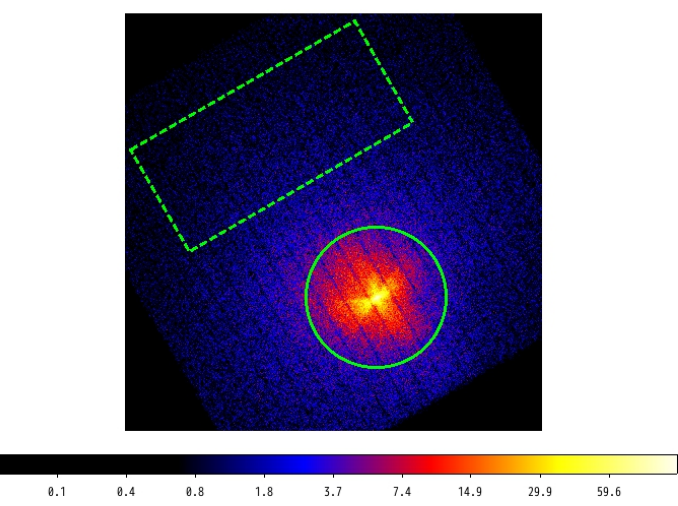

Figure 3: Extraction region of Suzaku spectra overlaid on the XIS1 image.The green circle with 3 arcmin is the source region, and the green box $(5 \operatorname{arcmin} \times 11 \mathrm{arcmin})$ is the background region for spectral analysis.

Intraday variability is seen in Suzaku observation in 2006 (Fig.4(b)) and Chandra observation in 2017 (ID:18612, Fig.4(a)). Suzaku light curve in 2006 showed approximately 30\% variability with a time scale of 0.3 days. For Chandra light curve of the core in 2017, count rate changed approximately $40 \%$ with a time scale of 0.5 days. However, since the extraction region of Chandra data was as small as 2.0 arcsec, the fluctuation of the satellite attitude may cause a variability of the count rate. Therefore, we made light curves with different extraction radii of $0.4,1.0,2.0$, and 4.0 arcsec to evaluate the systematic effects. From this analysis, RMS became $0.10 \pm 0.01,0.09 \pm 0.01$, $0.08 \pm 0.01$, and $0.06 \pm 0.01$, for $0.4,1.0,2.0$, and 4.0 arcsec, respectively. The RMS decreased as the radius increased, but variability was significant, even for a large radius.

\section{Discussion and summary}

The results obtained in this study are summarized below. First, the Suzaku observation in 2006 and the Chandra core observation in 2017 showed a significant intraday X-ray variability. In the Suzaku light curve, the variability amplitude was approximately $30 \%$ with a time scale of about 0.3 


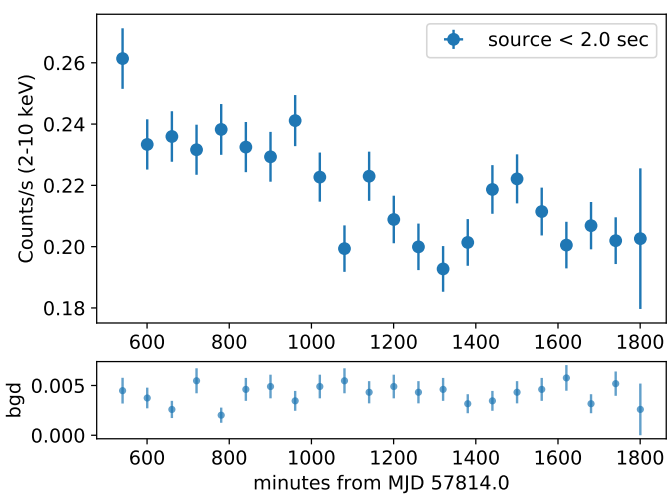

(a)

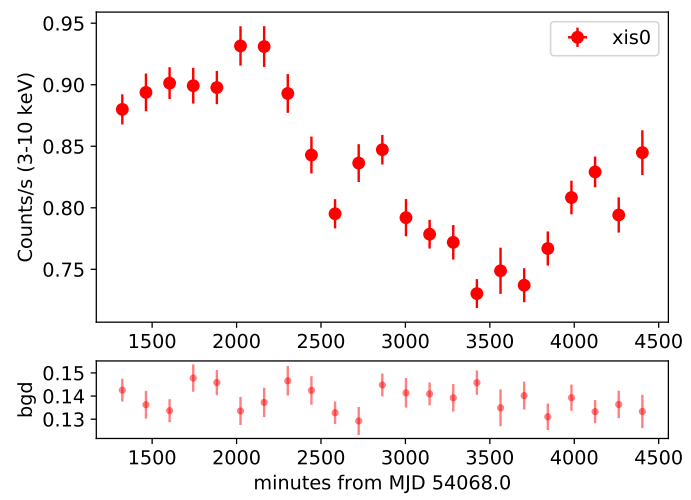

(b)

Figure 4: Light curves which showed fast variability taken by (a) Chandra core in March 2, 2017 (b)Suzaku/XIS0 in November 29, 2006.

days. For Chandra, the amplitude was approximately $40 \%$ with a time scale of 0.5 days. The HST- 1 was much brighter than the core in the X-ray band in 2006 [9]. On the other hand, the core was brighter than the HST-1 in 2017. The spectrum obtained by Suzaku in 2006 showed a steep photon index of $2.38_{-0.04}^{+0.07}$, and the core obtained by Chandra and NuSTAR in 2017 had a hard photon index of $1.96_{-0.04}^{+0.05}$. We estimated minimum size $R_{\min }$ of emission region by X-ray variability time scale $\Delta t$ as follows,

$$
R_{\min }=\frac{c \Delta t \delta_{D}}{1+z}
$$

where $\delta_{D}$ is a Doppler factor and $z$ is a redshift. We found $\Delta t \sim 0.3$ day in 2006, and thus we obtain $R_{\min } \simeq 1.0 \delta_{D} \times 10^{15} \mathrm{~cm}$. Here, a central black hole mass was assumed to be $M_{\mathrm{BH}}=(6.5 \pm 0.7) \times 10^{9}$ $M_{\odot}$ [3], The Schwartzschild radius is $R_{S} \sim 2.0 \times 10^{15} \mathrm{~cm}$. Thus, the size of the emission region was as compact as about $\sim R_{\mathrm{S}}$. Suzaku-XIS cannot resolve each component of the M87 jet, but core, knots D, and A cannot be a candidate of the source of this variability as follow reasons. Knot D and A were much fainter than HST-1 and their X-ray spectra were soft with a photon index of 2.4-2.6, $\simeq 2.2$, respectively [6]. Thus it is difficult for the knots $\mathrm{D}$ and A to explain a rapid variability above $3.0 \mathrm{keV}$.

For the core, from the result of Chandra on November 13, 2006, a count rate ratio of HST-1 to the core was approximately about 4.45 [9]. If the variability was only due to the core, A core count rate of the core must vary with an amplitude $\sim 260 \%$. Such an extreme large amplitude of variability has never been observed for the M87 core, and thus it is unlikely, we estimated this variability from HST-1.

[2] showed that X-ray emission observed by Suzaku could be explained by a high-energy tail of synchrotron, based on the spectral energy distribution (SED) modeling and thus concluded that HST-1 is likely the origin of TeV gamma-rays. From these results, X-ray intraday variability in 2006 observed by Suzaku-XIS can be explained by synchrotron radiation from HST-1.

Assuming that X-ray decay was due to electron cooling, we could estimate a magnetic field strength. The cooling time scale was expressed by the following equation from [7]

$$
\tau_{s}=3.2 \times 10^{4} \times B^{-3 / 2} \times E_{\mathrm{ph}}^{-1 / 2} \times \delta^{-1 / 2}(\mathrm{~s})
$$


where, $\tau_{s}$ is the decay time scale, $B$ is the strength of magnetic field, $E_{\mathrm{ph}}$ is the photon energy in the observer frame, and $\delta$ is the Doppler factor. Using a decay time scale of 0.3 days, and $E_{\mathrm{ph}}=6500 \mathrm{eV}$, and a Doppler factor $\delta$, the magnetic field became $B=1.94 \delta^{1 / 3} \mathrm{mG}$. Using the estimated magnetic field, we estimated the electron energy emitting synchrotron X-ray emission to, be $1.1 \times 10^{8} \gamma^{-1} \delta^{-1 / 3} \mathrm{TeV}$, and thus particle acceleration up to $\mathrm{TeV}$ was indicated to occur in the HST-1.

Next, we discuss about an X-ray variability of the core with a time scale $\Delta t \sim 0.5$ days in 2017. The X-ray flux of the core simultaneously varied with TeV gamma-ray flux in VERITAS observations in 2008. It suggesting that the core X-ray emission originated from the jet as well as TeV gamma-ray [1]. Therefore, the 2017 variability could be due to jet emission at the core. The power-law photon index of the core X-ray spectrum in 2017 is $1.96_{-0.04}^{+0.05}$, which could be the inverse Compton scattering component rather than synchrotron radiation if it comes from the jet. However, such a photon index can be explained by disk/corona or ADAF emission.

\section{Acknowledgement}

This research has made use of data and software provided by the High Energy Astrophysics Science Archive Research Center (HEASARC), which is a service of the Astrophysics Science Division at NASA/GSFC.

\section{References}

[1] Acciari, V. A., Beilicke, M., Blaylock, G., \& Bradbury, S. M. 2008, The Astrophysical Journal, 679, 397, doi: $10.1086 / 587458$

[2] de Jong, S., Beckmann, V., Soldi, S., Tramacere, A., \& Gros, A. 2015, Monthly Notices of the Royal Astronomical Society, 450, 4333, doi: 10.1093/mnras/stv927

[3] Event Horizon Telescope. 2019, The Astrophysical Journal Letters, 875, L6, doi: 10 .3847/ 2041-8213/ab1141

[4] Koyama, K., Tsunemi, H., \& Dotani. 2007, Publications of the Astronomical Society of Japan, 59, S23, doi: $10.109 /$ pasj/59.sp1.S23

[5] Shirai, H., Fukazawa, Y., Sasada, M., Ohno, M., \& Yonetoku. 2008, Publications of the Astronomical Society of Japan, 60, S263, doi: 10.1093/pasj/60.sp1.S263

[6] Sun, Xiao-Na, Yang, Rui-Zhi, Rieger, Frank M., Liu, Ruo-Yu, \& Aharonian, Felix. 2018, Astronomy and Astrophysics, 612, A106, doi: 10.1051/0004-6361/201731716

[7] Tashiro, M., Makishima, K., Ohashi, T., et al. 1995, PASJ, 47, 131

[8] Wong, K.-W., Nemmen, R. S., Irwin, J. A., \& Lin, D. 2017, The Astrophysical Journal, 849, L17, doi: $10.3847 / 2041-8213 /$ aa92c2

[9] Yang, S., Yan, D., Dai, B., et al. 2019, Monthly Notices of the Royal Astronomical Society, 489, 2685-2693, doi: $10.1093 / \mathrm{mnras} / \mathrm{stz} 2302$ 